\title{
Using Virtual Reality for Interpreter-mediated Communication and Training
}

\author{
Panagiotis D. Ritsos, Robert Gittins, Jonathan C. Roberts \\ School of Computer Science \\ Bangor University \\ Bangor, UK \\ $\{$ p.ritsos, r.gittins, j.c.roberts\}@bangor.ac.uk
}

\author{
Sabine Braun, Catherine Slater \\ Centre for Translation Studies \\ University of Surrey \\ Guildford, UK \\ $\{$ s.braun,c.slater\}@surrey.ac.uk
}

\begin{abstract}
As international businesses adopt social media and virtual worlds as mediums for conducting international business, so there is an increasing need for interpreters who can bridge the language barriers, and work within these new spheres. The recent rise in migration (within the $\mathbf{E U}$ ) has also increased the need for professional interpreters in business, legal, medical and other settings. Project IVY attempts to provide bespoke 3D virtual environments that are tailor made to train interpreters to work in the new digital environments, responding to this increased demand. In this paper we present the design and development of the IVY Virtual Environment. We present past and current design strategies, our implementation progress and our future plans for further development.
\end{abstract}

Keywords-Virtual Worlds, Interpreting, Second Life, Immersion, User Experience

\section{INTRODUCTION AND MOTIVATION}

Nowadays, interpreters are being called to master an ever broadening range of interpreting scenarios and skills, often required to help in demanding situations such as courts, police interviews and medical emergencies. However, training for such situations can be challenging to do through traditional teaching methods. In addition, efforts to educate potential clients of the realities of working with an interpreter are scarce and normally separate from interpreter education.

In this paper we present the IVY Virtual Environment (IVY-VE). The aim of IVY is to address these needs, offering future interpreters (trainees) and future users of interpreters (clients) a 3D virtual environment that supports the acquisition and application of skills required in interpreter-mediated communication, focusing on the practice of consecutive and liaison interpreting in business and community settings. In contrast to many other initiatives in interpreter training, IVY attempts to integrate the education of interpreters and their clients closer together by employing working modes which allow the two user groups to engage with the environment in a variety of ways.

The specific aims of project IVY are to develop a) an adaptive 3D virtual environment for interpreting students and future users of interpreters; b) a range of virtual interpreting scenarios (e.g., 'business meeting') that can be run in different modes (e.g., 'interpreting mode', where interpreting students can practice using dialogues and monologues as shown in Figure 1; 'exploration mode', where clients can learn about interpreting; 'live interaction', where both groups can engage in role plays); c) integrate into the virtual environment multilingual audiovisual content based on video corpora from the LLP project BACKBONE [1]; d) create two sets of learning activities, one for students and one for clients (e.g., interpreting and awareness-raising exercises).

IVY-VE supports these aims by fusing web-based technologies with Second Life. Through the use of web technologies we offer intuitive means of managing scenarios and resources within the $3 \mathrm{D}$ environment. Our implementation approach follows an Agile methodology [2], evaluating our prototypes in iterations. For that purpose we present a critical evaluation of our prototype as well.

The structure of the paper is as follows: Section II presents related work of training in Virtual Worlds, ICTbased interpreting training and the use of web technologies with virtual worlds, Section III describes several alternative design strategies that we considered, Section IV explains in detail our implementation, Section $\mathrm{V}$ reports on the findings of a preliminary evaluation, Section VI discusses our prototypes current limitations and Section VII summarizes our conclusions and presents our future plans.

\section{Previous Related Work}

Today's virtual worlds and massively multiplayer online role-playing games (MMORPGs) are descendants of Multiuser virtual environments (MUVEs) [3], [4] of the 1980s. Current examples, such as Second Life (secondlife.com) or World of Warcraft (us.blizzard.com) appear to have a strong appeal on users [5], [6] and seem to enhance participant involvement when used for educational and training purposes [7]. A comprehensive presentation of virtual worlds, their history and availability to date is beyond the scope of this paper; the reader can obtain such information from other sources such as Bartle [4] and Duncann et al. [8]. Instead, we focus on the most prominent examples, employed in education and training.

Second Life (SL) is a freely accessible 3D multi-user, collaborative, virtual world developed by Linden Labs, with millions of subscribers throughout the world. It is arguably 


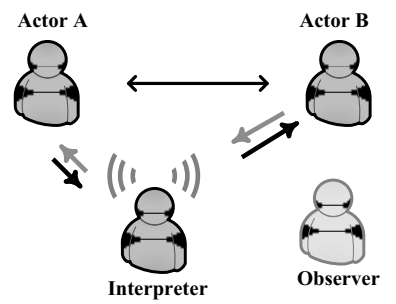

(a) Dialogue scenario

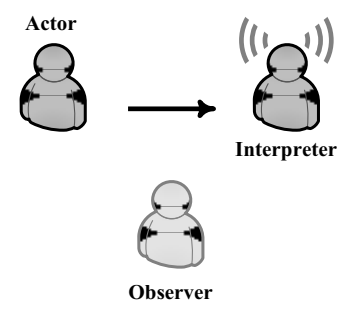

(b) Monologue scenario
Figure 1. A range of scenarios is created in IVY VE to support interpreting practice in ready-made dialogues (a) and monologues (b) as well as live interaction and other working modes. In the dialogues and monologues, the actors are avatars controlled by the environment (robots), representing the interlocutor(s) in each scenario, and the avatar of the interpreting student. In live interactions, avatars of interpreting students and their 'clients' interact.

the most popular commercial virtual world, with 170 accredited educational institutions represented in 2008, as reported by Jennings and Collins [9] and used in various fields such as medical and health education [10], environmental education [11] and security [12]. Another virtual world that has attracted the attention of researchers [13]-[15] is Activeworlds (activeworlds.com) launched in 1997 which includes a dedicated educational world, called Educational Universe (AWEDU). OpenSimmulator (opensimulator.org) is a newer virtual world implementation, in the form of an open source multi-platform, multi-user 3D application server. It is almost exclusively used by universities and big companies and has attracted the attention of researchers of newer efforts, such as the one described by Zhao et al. [16] and Konstandinidis et al. [17].

\section{A. ICT-based interpreter training}

While virtual worlds have not been adopted in interpreting training programs, other Information and Communication Technologies (ICT) have been successfully used. Not only do these systems allow users to practice, but using them also helps trainee interpreters develop computing literacy, which is becoming increasingly important in the interpreter's daily work [18].

The SIMON (Shared Interpreting Materials Online) project [19] was designed to complement the Virtual Institute at the Ecole de Traduction et d'Interprétation at the University of Geneva and is an online resource for interpreter trainers. The material is classified according to progression level, exercise type language and thematic domain.

Hansen and Shlesinger [20] describe a video-based interpreter training initiative, used at the Copenhagen Business School, where a series of video clips, in which tutors and others perform monologues and bilingual role-play dialogues. Similarly, the English Language Interview Corpus as a Second-Language Application (ELISA) [21] is a collection of video interviews with native speakers of English which is designed to be a resource for language learning and interpreter training. ELISA was a forerunner for BACKBONE [1] which produced video interviews with native speakers of English, French, German, Polish, Spanish and Turkish, as well as with non native speakers of English. The BACKBONE resource also includes interpreting exercises that can be applied to any of the videos in the BACKBONE corpora.

Sandrelli and Jerez [22] describe the evolution of authoring programs for interpreters, starting with Interpr-IT, through Interpretations, and finally to Black Box 3.0. They highlighting functionalities such as a voice recording feature, on-screen note-pad, pitch-tracker for gauging performance etc. While authoring options go beyond the IVY environment, some similar tools may be integrated in the future.

Tymczynśka [23] describes how the Moodle Learning Management system [24] was integrated into a healthcare interpreting module at Adam Mickiewicz University in Poland. She highlights five different criteria for evaluating the applicability of the learning resources — student learning potential, student and context fit, authenticity, practicality and sense of achievement. These are similar to the findings from the pedagogical considerations emerging from other virtual world projects.

\section{B. Pedagogy of Education and Training in Virtual Worlds}

The pedagogic implications of using Virtual Worlds in training and simulation scenarios have been investigated by many researchers. Savin-Baden et al. [25] argue that development in virtual environments, in the past, has led the pedagogy to date and that further development needs to be driven by pedagogy itself. Thackray et al. [26] point out that different methodologies among institutions, disciplines and instructors introduce great variety on virtual world implementations and associated practices and that there are inherent usability challenges of virtual environments amplifying those of the learning and teaching experience.

More importantly, Salmon et al. [27] point to the need to introduce students into the virtual worlds through familiarization sessions and the need to enhance their feeling of immersion through involvement with their avatars before they are asked to perform any significant tasks. Particularly in relation to Second Life, there is a steep learning curve in becoming familiar with the environment and its native functionality, increasing the challenges inherent to the learning process [28]. Tasks structure and intuitive confirmation of task outcomes can also be an important aspect, considering that Second Life can be chaotic and anarchic, as claimed by Carr et al. [28]. In addition, Moschini [29] highlights the fact that users of such environments expect interactive content, rather than a mere passive learning experience, becoming active participants.

It is interesting to note, nonetheless, that none of the studies reported have researched whether the ability to learn improves in virtual worlds. An equally important aspect is 
that studies to date have predominately focused on students in a higher education environment.

\section{Web technologies and Virtual worlds}

One of the drawbacks of most virtual environments is that they are exclusive [30] and often require 'in-world' scripting, even for the simplest examples of communication and mediation. Managing content in these worlds often requires knowledge of proprietorship scripting languages — such as LSL (Linden Scripting Language) used in Second Life which arguably do not have the features of established web technologies like Java, XML etc. and are often not flexible when it comes to implementations requiring frequent content management and updating.

Some researchers have investigated how virtual worlds and such web technologies merge [31] to allow for better content management and in-world presentation. An example of fusing Second Life with web technologies, is Sloodle, a Second Life fusion with Moodle [24], presented by Kemp and Livingstone [32]. Sloodle presents information in Second Life using HTTP-requests, communicating with Moodle's PHP-based database front end. Zender et al. [30] present their SOA-based approach, using Web Services to provide lecture streaming and a messaging service in Second Life.

Nonetheless, there are important limitations in establishing communication between Second Life and external entities (e.g., scripts, programs). LSL uses functions that utilize HTTP and XML, such as llHTTPRequest or the older RPC-based lisendRemoteData and llRemotedataReply for this purpose. However, Linden imposes time delays and limits on these methods, such as request size, number of requests per object, per owner and per period of time, making more demanding communication tasks quite difficult to implement.

\section{Design Strategies}

The first strategic choice concerned the virtual world to be used. The main advantages that led to using Second Life for the IVY project prototypes, when compared to other virtual worlds (such as OpenSimulator) are:

- SL provides a large community of users, developers and enthusiasts. These users have created many addons, plugins and therefore there are many examples of customizations and vast support for scripting and assets for creating the scenario settings.

- SL is a platform for social interaction and education, used by numerous institutions, colleges, universities thus through using SL it will increase the chances of exposure and information dissemination of the IVY project.

- SL does not require that users run the virtual world themselves, but can access public servers; subsequently, prototypes could be quickly built and shared between the developers and partners in IVY.

Bearing in mind the decision to use Second Life two alternative implementation strategies were considered. The main decision was whether the IVY-VE interface would be scripted in Second Life, using LSL or implemented as a separate, external component using popular web technologies such as HTML/CSS and Java.

The major advantage of the first solution is the fairly high level of integration of the interface with the SL client's GUI. Generally menus and GUI elements built using LSL have comparable aesthetics to the main client GUI and appear crisper and clearer. Embedding scripts into objects is a common practice in the virtual world and many information display systems make use of such mechanisms. Nonetheless, due to the static nature of scripting data into scripts dynamic updating of the menu content becomes quite tedious. LSL has support from connecting to a database, most commonly through PHP scripts, as used in some research efforts described, but it is quite limited in terms of the size of information that can be sent or received. Overall, this solution currently offers limited capabilities compared to HTML/CSS and Java-based web interfaces.

The second approach has the advantage of relying on popular and established techniques for building web applications, far more flexible than LSL. The level of menu customization and enrichment, database connectivity and overall flexibility surpasses that of LSL-based scripting. However, this implementation requires either embedding an SL viewer on a web page or an web-like, in-world interface. Unfortunately, despite the popularity of the notion of webbased SL viewers, Linden Labs does not officially offer a web client ${ }^{1}$ and most of the efforts to implement something similar seem to have ceased. Canvas, from Tipodean Technologies (tipodean.com) appears to be the only implementation of such a viewer, claiming to support both SL and OpenSim.

\section{IMPLEMENTATION STRATEGY}

Our chosen implementation strategy aspires to merge the useful features from both alternatives, resulting into a hybrid solution. One module consists of a web application with two entry points. One entry point remains independent of SL and is used from content managers to manage scenarios and user's information, offering basic CRUD functionality. The other end is viewable within Second Life, in the form of a 'Heads-up-Display' (HUD), populated from a database, showing available scenarios to the users, having a player functionality and initiating in-world teleport events. Figure 2 depicts the system architecture.

Due to limits on the number of primitives available to the IVY Island and the lack of instancing mechanisms in SL (see

\footnotetext{
${ }^{1} \mathrm{~A}$ beta version of a web-based client has been made public but its current state is unknown
} 


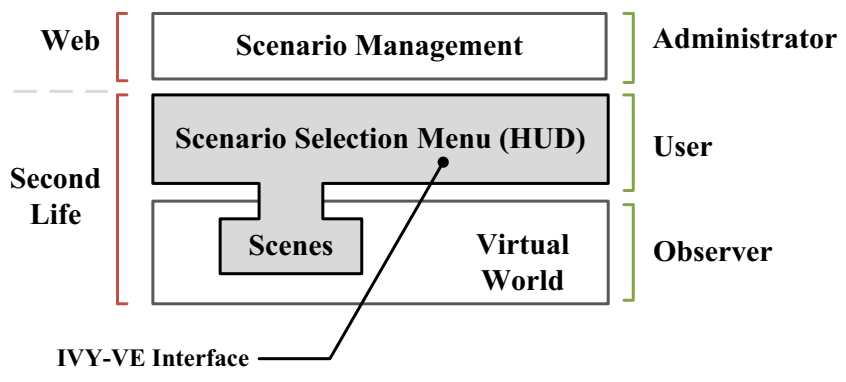

Figure 2. IVY-VE Architecture - depicting the three main domains of the application, namely the web-based administration panel, the in-world HUD, the scenes associated with the scenarios and the remaining, surrounding virtual world

Section VI) IVE-VE uses a collection of unique, in-world locations for each type of scenario (e.g., Classroom, Meeting Office). Therefore, a scenario may share its location with another, being carried out in a similar setting. In order to maintain consistency in the virtual world only one scenario can be played per location at a given time. Upon a scenario launch by a user, all scenarios sharing the same location become unavailable for other users. Once the user exits the selected scenario, all scenarios sharing the same location become available again. At this stage, it was preferred to maintain consistency in the virtual environment and explore scalability in future implementations. Figure 3 shows the relationships and sequence of actions in IVY-VE.

\section{A. User Classes and Characteristics}

Separating user roles allows controlled access to different parts of the application as well as means of monitoring scenario selection and execution. The system's user classes and their respective role descriptions are:

i) Interpreters/Users, whose purpose is to explore, participate and exercise with the scenarios in Second Life

ii) Content Managers who are responsible for user and scenario management.

iii) Observers, whose purpose is to observe other users in Second Life.

\section{B. The IVY Island}

The IVY environment was built in SL using several geometric models and textures. Photorealistic landscaping of both immediate scenario locations and distant views provided a generic setting for the project, and a series of locations including a reception area, a meeting room, a classroom and an office have been built. The goal throughout was to use the smallest number of primitives to allow scalability and replication in the future. The reception area serves as the central hub of movement for visitors where they can obtain the HUD, view introductory videos and read noticeboards with information. Care and effort has been put into the scale of objects, in order to create a realistic environment, following a course of action as the one described by Roberts et al. [33]; Figure 4 shows some of the locations.

\section{Scenario Actors}

We populate the scenarios with additional 'actors'. Currently these actors are manually placed in each location, using Second-life 'robots'. These 'bots' are from Pikkubot (pikkubot.de) and Thoys (slbot.thoys.nl). In our implementation each bot is controlled from a separate server, either using in-world chat or command lines in the server's telnet prompt. The ultimate aim is for the bots to relay sound when it is their turn to speak and perform gesture animations related to the scenario. Currently the bots use some recursive animation overrides (AO) that make them appear life-like.

\section{The IVY web application system}

The IVY web application was build using the Appfuse 2 open source project (appfuse.org). Appfuse, built on the Java platform, uses industry-standard features, such as Apache Maven integration, JPA support for database operations and popular web frameworks such as Spring and Apache Struts, employed in this example. Our prototype is deployed using Apache Tomcat 6.x and uses the MySql 5.x database.

\section{E. Audio File Format, Segmentation and Management}

The IVY-VE uses audio extracts (segments), in MPEG-2 Audio Layer III format, from the LLP project BACKBONE, wrapped in XSPF (XML Shareable Playlist Format) [34] play-lists (scripts) and played within pre-fabricated scenes. Audio segments are uniquely named and can be interchanged - within each script - to derive further language combinations of that scenario. Actors may speak for more than one consecutive segments, allowing greater flexibility in creating scripts where one talks for extended periods. However, it is assumed that only one actor talks per audio segment and there is no overlap between actor's speech. Each script has textual information associated with it, such as brief content information, scene description and domain keywords.

\section{F. In-World Heads Up Display}

The HUD makes use of the 'HTML-on-a-prim' feature of Second Life, where HTML can be mapped on virtual objects (boards, panels etc). Audio is being played by means of a Flash player, parsing the XSPF playlists upon dialogue selection. Navigation through the island is performed using slurls, providing direct url-like teleport links to locations within the virtual world. Each slurl is being called upon scenario launch, triggering the native SL-client teleport interface.

The HUD (Figure 5) is built using the jQuery JavaScript library, displaying list of the scenarios in database as a drilldown menu in small footprint and placed at the lower left of the user's viewport. Upon selecting a scenario one can read the scenario information, launch the scenario player 


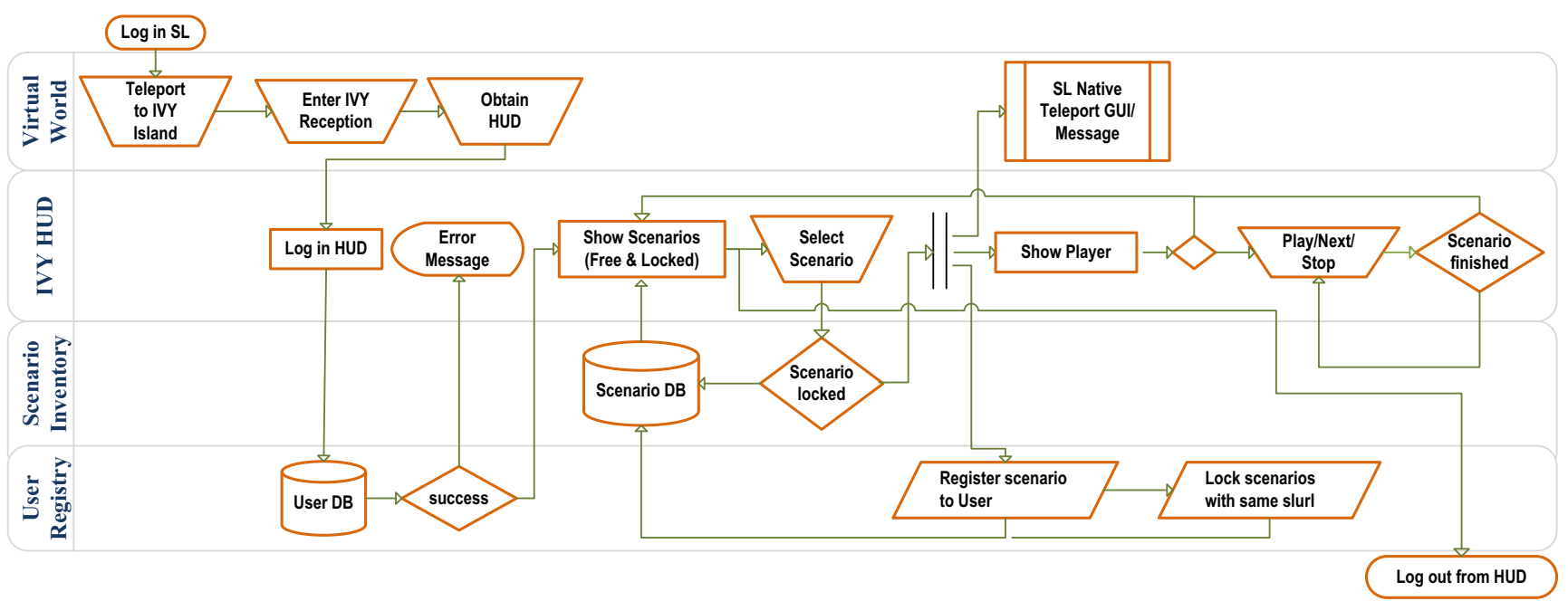

Figure 3. IVY-VE Cross-functional flowchart - depicting the functions and the process flow, in each tier of the application, from log-in to dialogue selection and playback.

and trigger the teleport interface of SL. Figure 4 shows some screenshots of the IVY-VE.

\section{G. Administrator's Panel}

One of the most important functionality features of the IVY-VE is the scenario and user management console. The use of Appfuse simplified the implementation of features related to internationalization, security, database integration, user management and offered a basic CRUD backend. This was further enhanced with tools to create, edit and inspect scenarios and monitor their usage.

Content administrators can create new scenarios, through a form (shown in Figure 6) where they provide textual information (title, language combinations, participants gender etc.) as well as an ordered file listing of the audio tracks of each scenario. Textual information is stored in the database and the audio file listing in XSPF format, in the playlist inventory.

The scenario-listing view allows content administrators to monitor the usage of their dialogues, depicting which user is currently working on each scenario and which scenarios, sharing the same location, are unavailable at a given time. Administrators can choose to use the slurl links to launch their SL client and teleport to those locations. Particular attention was paid to extended language support, so that scenario information in different character sets can be entered and displayed properly.

\section{Preliminary Functional Evaluation}

As part of the Agile methodology we follow in the development of IVY-VE, our prototype has gone through various iterations of evaluation. Following the feasibility assessment of the aforementioned design alternatives a preliminary evaluation of the current prototype was done by interpreting and virtual world experts. This evaluation attempted to highlight problems and useful features of our design.

The evaluation was done using talk-aloud, try-out sessions in Second Life, followed by a discussion with the assessor and focused on the in-world HUD and scenario settings. The administrator's panel was not evaluated at this stage; however, a more structured functional evaluation will follow in the forthcoming months, inspecting that functionality as well. Nine experts in Interpreting and two in Virtual Worlds used the HUD for brief sessions of 10-15 minutes in the IVY environment. Feedback was collected by the authors and comments and observations were recorded and collated after the try-out sessions.

One of the most important outcome of the discussions was that the users focused more on the player rather than on the scenario and the actors. Despite that observation, most users felt it is simple enough to execute the scenarios. However some felt that the fact that observers in SL can only listen the interpreter, but not the actors, is a limitation.

Scenarios that were in use by other users were locked. In the test environment, these locked scenarios were not shown in the HUD at the time and some participants, although acknowledged the virtual worlds limited scalability implications, requested that all scenarios would be nonetheless displayed, and shown as being 'in use'. This feature was subsequently implemented, as shown in Figure 5. Another request was to make the HUD bigger and movable. Most uses commented favorably on the realism and aesthetics of the virtual world locations and all appeared satisfied with the scenario selection and teleportation mechanism.

Overall the interpreter experts were positive on seeing the system become operational and expressed their confidence that it can develop into a tool they would be willing to apply in their practice. The IVY-VE is currently being extended 


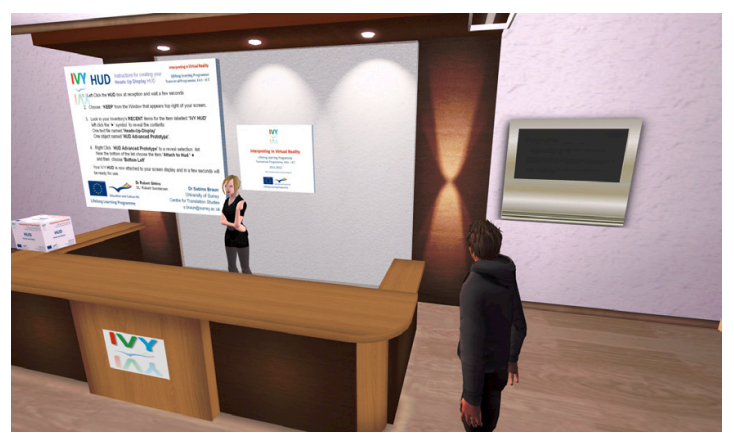

(a) IVY Reception

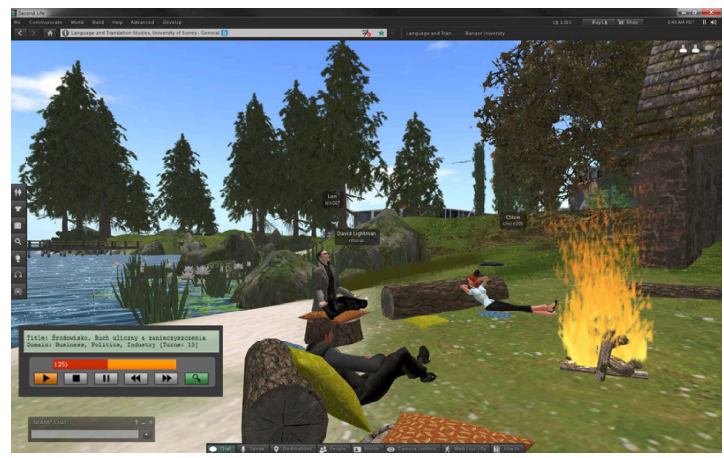

(c) Outdoor setting

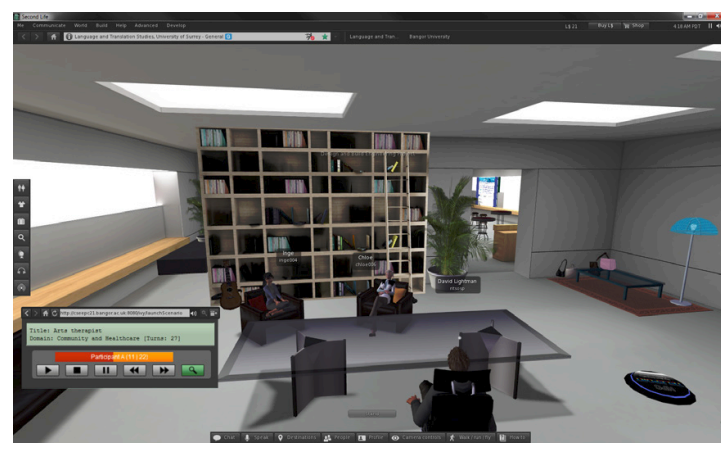

(b) Arts Therapy Room

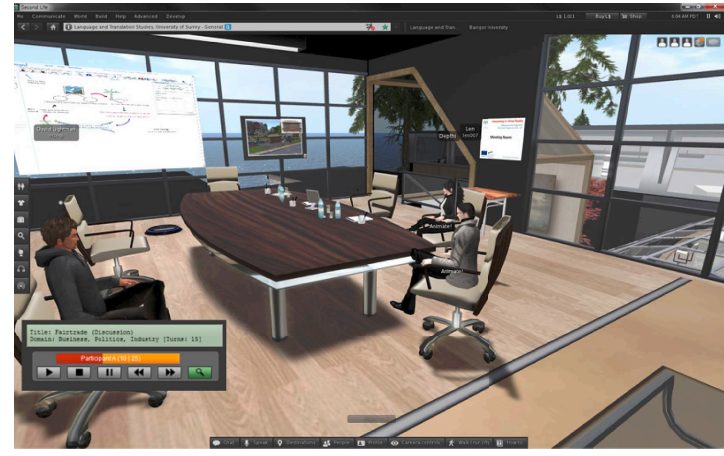

(d) Meeting Room

Figure 4. Screenshots of the IVY-VE in use, showing scenario locations in Second Life - The IVY Reception (a) is where visitors are initially teleported and where they pick the HUD from. The locations where designed to match the available scenario themes, maintaining a generic look as much as possible to allow re-use of primitives

and a wider trial with interpreting students and clients is planned.

\section{LimitATIONS AND FUTURE WORK}

In an effort to continuously amend our implementation we have classified limitations and problems, of our current prototype, in four categories: virtual world scalability, scenario management, actor management and scenario execution.

Virtual world scalability issues occur mainly because we are limited in number of objects ('prims') we can create in the SL island. At the moment we use unique model locations for all scenarios of a particular setting. Executing one scenario essentially blocks other users from using a scenario at that location, in order to maintain consistency in the virtual world. The game world has dealt with similar problems, applying the concepts of zoning, instancing and replication. Such design concepts are difficult to achieve in SL and thus these design ideas may suggest that a different infrastructure to one based on Second Life may be a more appropriate solution. On the other hand, SL has enabled us to create a useful and working system in a short time and with little cost. For the time being we are experimenting with replicating models of each setting and using a location registry to assign them in each scenario.
Scenario management, in terms of the availability at a given time, is consequently affected by the scalability issue.

Actor management is also associated to the scalability issue. Currently, robots are manually placed in each scenario. Automatic robot placement into each scenario's location is under development, however this is a difficult challenge and we are currently experimenting with two approaches: as part of the web application and in-world.

One approach is to enhance our system with separate service components that will control the 'bots', resulting in a SOA-like architecture. Our version does not employ at this stage any such units but it has been designed with provisioning to accommodate them in the future. However, this directly affects the scenario execution, as the service units controlling the actors need to be directly connected to the player to enable on-demand playback - much like a puppeteer (interpreter) controlling the puppets ('bots'actors).

An alternative scheme is to attach in-world players in each scenario setting and modify the audio of the player upon scenario launch, also having consequences on scenario management and scenario execution. This may also ameliorate the issues reported during the evaluation that using the HUD seems to disrupt the sense of immersion experienced 


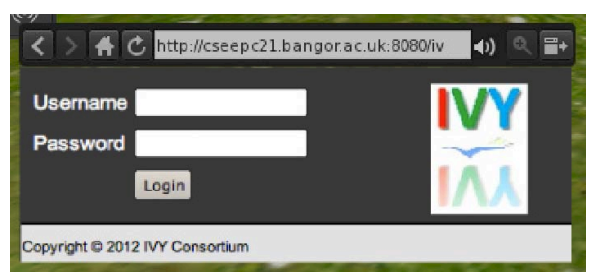

(a) Login Screen

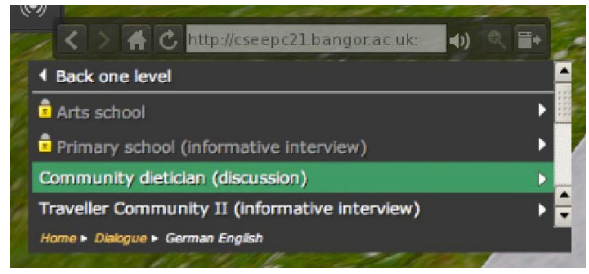

(b) Title listing with locked scenarios

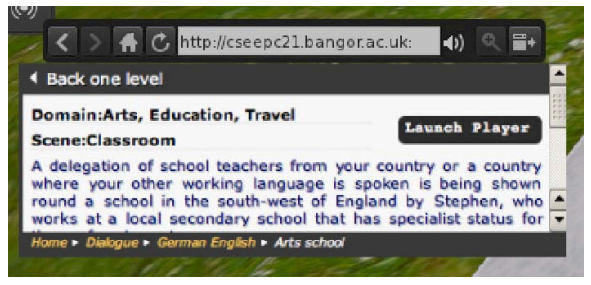

(c) Scenario Details

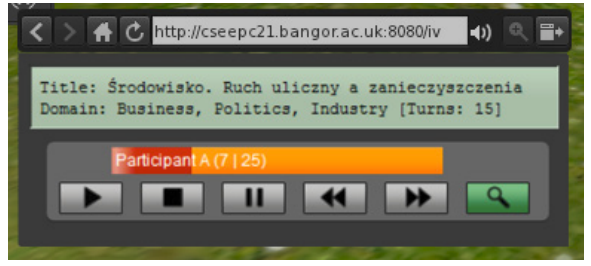

(d) Scenario player

Figure 5. Screen captures of the IVY-VE HUD, depicting the login screen (a), the title selection view with some of the scenarios locked (b), the scenario inspection pane (c), with textual information and launch button and the scenario player (d)

by users. In addition, we are also keen on providing some form of basic animation on our robots, synchronized to the content of each scenario.

Furthermore, one important limitation is that the sound of the scenario actors, played from the IVY-VE player, is heard only by the user but not by observers, as it is not broadcasted back to the SL-location. In addition, from the interpreters point of view, the sound does not appear to originate from each actor as there is no sound directionality and no visual cues, such as flashing of the actor's overhead voice-intensity indicator to denote who is speaking.

Some of the limitations, described above, could be overcome by developing a bespoke virtual system such as by using the Unity game engine (unity3d.com). This could integrate sound, virtual movement, robot interaction and inworld communication in a single application. Indeed other

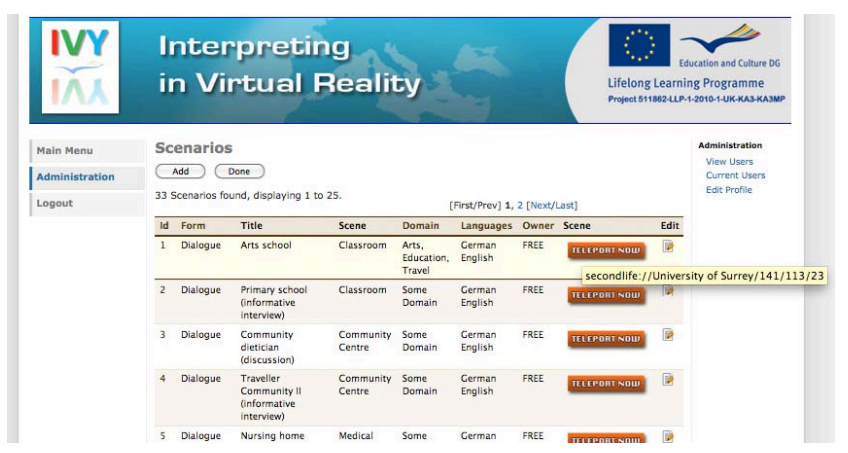

Figure 6. The Administrator's panel includes tools to create, edit, delete scenarios and users as well as monitor scenario usage, launch SL client and teleport to scenario locations, using slurls.

modalities could be added into the world (such as haptics [35]) to develop a more realistic interactive experience. Our aspiration is to explore a self-made solution once we have investigated the associated user experience of interpreter students and clients, through the current generation of prototypes.

\section{CONCLUSIONS}

In this paper we present the IVY-VE, to design, implement and evaluate a $3 \mathrm{D}$ virtual environment that supports the acquisition and application of skills required in interpretermediated communication. The system is based on the fusion of Second Life and web technologies such as Appfuse, jQuery and JavaScript. Our approach benefits from managing the audio content as a web service while exploiting the benefits if using 3D content creation and user exploration in a public 3D world such as SL. This separation also enables different 3D worlds to be used instead of Second Life (such as OpenSimulator) in the future.

We have performed a functional evaluation, that was tested on Interpreting and Computer Science experts, and we elicited potentially useful features and limitations. We have identified and discussed these issues and provided a description of our future plans to rectify problems and enhance overall user experience of the system.

In conclusion we have developed a usable virtual environment for interpreting students and clients, and our early evaluation of the tool suggests it to be useful and valuable. However more work is required to develop IVY further and incorporate additional scenarios, and evaluate the system with interpreting students and clients.

\section{ACKNOWLEDGEMENTS}

We are grateful to the members of the IVY consortium, for their assistance and contribution with the scenario material and their feedback during the evaluation. This work was supported by the European Commission through project 511862-LLP-1-2010-1-UK-KA3-KA3MP in the Lifelong Learning Programme. 


\section{REFERENCES}

[1] K. Kohn, P. Hoffstaedter, and J. Widmann, "BACKBONE - Pedagogic Corpora for Content \& Language Integrated Learning," in Eurocall Conference Proceedings. Macmillan ELT, 2009.

[2] J. Highsmith, Agile software development ecosystems. Addison-Wesley Longman Publishing Co., Inc., 2002.

[3] S. Warburton, "Second Life in higher education: Assessing the potential for and the barriers to deploying virtual worlds in learning and teaching," British Journal of Educational Technology, vol. 40, no. 3, pp. 414-426, 2009.

[4] R. Bartle, "From muds to mmorpgs: The history of virtual worlds," International Handbook of Internet Research, pp. 23-39, 2010.

[5] A. deNoyelles and K. K.-J. Seo, "Inspiring equal contribution and opportunity in a 3D multi-user virtual environment: Bringing together men gamers and women non-gamers in Second Life," Computers \& Education, vol. 58, no. 1, pp. $21-29,2012$.

[6] N. Yee, "The demographics, motivations, and derived experiences of users of massively multi-user online graphical environments," Presence: Teleoperators and virtual environments, vol. 15, no. 3, pp. 309-329, 2006.

[7] L. Jarmon, T. Traphagan, M. Mayrath, and A. Trivedi, "Virtual world teaching, experiential learning, and assessment: An interdisciplinary communication course in Second Life," Computers \& Education, vol. 53, no. 1, pp. 169-182, 2009.

[8] I. Duncan, A. Miller, and S. Jiang, "A taxonomy of virtual worlds usage in education," British Journal of Educational Technology, 2012.

[9] N. Jennings and C. Collins, "Virtual or Virtually U: Educational Institutions in Second Life," International Journal of Social Sciences, vol. 2, no. 3, pp. 180-186, 2007.

[10] M. Boulos, L. Hetherington, and S. Wheeler, "Second Life: an overview of the potential of 3D virtual worlds in medical and health education," Health Information \& Libraries Journal, vol. 24, no. 4, pp. 233-245, 2007.

[11] E. Ye, Y. Fang, C. Liu, T. J. Chang, H. Q. Dinh, and I. Design, "Appalachian Tycoon: an environmental education game in Second Life," Electrical Engineering, pp. 72-76, 2007.

[12] J. Ryoo, A. Techatassanasoontorn, and D. Lee, "Security education using Second Life," IEEE Security Privacy Magazine, vol. 7, no. 2, pp. 71-74, 2009.

[13] K. Ang and Q. Wang, "A case study of engaging primary school students in learning science by using Active Worlds," in Proceedings of the First International LAMS Conference 2006: Designing the Future of Learning, 2006.

[14] M. Dickey, "Three-dimensional virtual worlds and distance learning: two case studies of Active Worlds as a medium for distance education," British Journal of Educational Technology, vol. 36, no. 3, pp. 439-451, 2005.

[15] E. Prasolova-Førland, "Analyzing place metaphors in 3D educational collaborative virtual environments," Computers in Human Behavior, vol. 24, no. 2, pp. 185-204, 2008.

[16] H. Zhao, B. Sun, H. Wu, and X. Hu, "Study on building a 3D interactive virtual learning environment based on OpenSim platform," in Audio Language and Image Processing (ICALIP), 2010 International Conference on. IEEE, 2010, pp. 1407-1411.

[17] A. Konstantinidis, T. Tsiatsos, S. Demetriadis, and A. Pomportsis, "Collaborative learning in opensim by utilizing sloodle," in Telecommunications (AICT), 2010 Sixth Advanced International Conference on. IEEE, 2010, pp. 90-95.
[18] C. Collins, "Looking to the future: Higher education in the Metaverse," Educause Review, vol. 43, no. 5, pp. 51-63, 2008.

[19] K. Seeber, "SIMON: An online clearing house for interpreter training materials," Technology and Teacher Education Annual, vol. 4, p. 2403, 2006.

[20] I. Hansen and M. Shlesinger, "The silver lining: Technology and self-study in the interpreting classroom," Interpreting, vol. 9, no. 1, pp. 95-118, 2007.

[21] S. Braun, "ELISA-a pedagogically enriched corpus for language learning purposes," Corpus Technology and Language Pedagogy: New Resources, New Tools, New Methods, 2006.

[22] A. Sandrelli, M. Jerez et al., "The impact of Information and Communication Technology on interpreter training," The Interpreter and translator trainer, vol. 1, no. 2, pp. 269-303, 2007.

[23] M. Tymczynska, "Integrating in-class and online learning activities in a healthcare interpreting course using Moodle," The Journal of Specialised Translation, no. 12, pp. 148-164, 2009.

[24] M. Dougiamas and P. Taylor, "Moodle: Using learning communities to create an open source course management system," in Proceedings of world conference on educational multimedia, hypermedia and telecommunications, vol. 3, 2003.

[25] M. Savin-Baden, L. Gourlay, C. Tombs, N. Steils, G. Tombs, and M. Mawer, "Situating pedagogies, positions and practices in immersive virtual worlds," Educational Research, vol. 52, no. 2, pp. 123-133, 2010.

[26] L. Thackray, J. Good, and K. Howland, "Learning and teaching in Virtual Worlds: Boundaries, challenges and opportunities," Researching Learning in Virtual Worlds, pp. 139-158, 2010.

[27] G. Salmon, M. Nie, and P. Edirisingha, "Developing a fivestage model of learning in Second Life," Educational Research, vol. 52, no. 2, pp. 169-182, 2010.

[28] D. Carr, M. Oliver, and A. Burn, "Learning, teaching and ambiguity in virtual worlds," in Learning in Virtual Environments International Conference, 2008, p. 83.

[29] E. Moschini, "The Second Life Researcher Toolkit-An exploration of in-world tools, methods and approaches for researching educational projects in Second Life," Researching Learning in Virtual Worlds, pp. 31-51, 2010.

[30] R. Zender, E. Dressler, U. Lucke, and D. Tavangarian, "Pervasive media and messaging services for immersive learning experiences," in Pervasive Computing and Communications, 2009. PerCom 2009. IEEE International Conference on, march 2009, pp. $1-6$.

[31] C. Blais, D. Brutzman, D. Horner, and M. Nicklaus, "Webbased 3D technology for scenario authoring and visualization: The SAVAGE project," in The Interservice/Industry Training, Simulation \& Education Conference (I/ITSEC). NTSA, 2001.

[32] J. Kemp, D. Livingstone, and P. Bloomfield, "SLOODLE: Connecting VLE tools with emergent teaching practice in Second Life," British Journal of Educational Technology, vol. 40, no. 3, pp. 551-555, 2009.

[33] J. C. Roberts, R. Gittins, and R. Thomas, "Scale and the construction of real-world models in second life," Journal of Gaming \& Virtual Worlds, vol. 2, no. 3, pp. 259-279, 2010.

[34] L. Gonze, M. Friedrich, and R. Kaye, "XML shareable playlist format version 1," 2008. [Online]. Available: http://www.xspf.org/xspf-v1.html

[35] S. A. Panëels and J. C. Roberts, "Review of designs for haptic data visualization," IEEE T. Haptics, vol. 3, no. 2, pp. 119137, 2010. 